\title{
Carcinoma verrucoso plantar. A propósito de un caso poco frecuente
}

\author{
Plantar Verrucous Carcinoma. Report of a rare case
}

\author{
Daniel López López ${ }^{1}$, David Rodríguez SAnz², Ángel Morales PoncEª \\ Alfredo Soriano Medrano ${ }^{4}$ \\ ${ }^{1}$ Doctor por la Universidade da Coruña. Profesor Asociado \\ Departamento de Ciencias da Saúde. Facultad de Enfermería y Podología. Universidade da Coruña. \\ daniellopez@udc.es \\ ${ }^{2}$ Doctor por la Universidad Rey Juan Carlos. Profesor Universidad Europea de Madrid. \\ davidrodriguezsanz@hotmail.com \\ ${ }^{3}$ Doctor por la Universidad Rey Juan Carlos. Clínica Podológica Torrijos, Toledo, España. \\ clinicatorrijos@gmail.com \\ ${ }^{4}$ Doctor por la Universidad Rey Juan Carlos. Clinisalud Centro Médico, Albacete, España. \\ soriano.alfredo@gmail.com
}

\begin{abstract}
Correspondencia:
Dr Daniel López López

Universidade da Coruña

Departamento de Ciencias da Saúde

Facultad de Enfermaría e Podoloxía

Campus Universitario de Esteiro s/n

E-15403 Ferrol

Correo electrónico: daniellopez@udc.es
\end{abstract}

Fecha de recepción: 2 de junio de 2012

Fecha de aceptación: 21 de diciembre de 2012

Los autores declaran no tener ningún tipo de interés económico o comercial.

\section{RESUMEN}

Antecedentes: Los carcinomas verrucosos en pocas ocasiones se mencionan en la literatura su localización en la planta de los pies, siendo una variante bien diferenciada del carcinoma de células escamosas visto en mucosa y piel, incluyendo los pies.

Objetivo: Describir las características clínicas, histopatológicas y de laboratorio y los resultados de un caso clínico inusual de un carcinoma verrucoso en la planta del pie.

Métodos: Presentamos un extraño caso de un carcinoma verrucoso en la planta del pie izquierdo, a nivel del primer metatarsiano, que fue resecado completamente y se quedó sin evidencia de recidiva tumoral o metástasis, durante el seguimiento en un periodo de 4 años. Sobre esta base, se revisaron los casos publicados de carcinoma verrucoso en el pie, para definir las peculiaridades de este tumor poco común y para dar recomendaciones diagnósticas y terapéuticas.

Resultados: El carcinoma verrucoso se caracteriza por una presentación clínica lenta pero inexorablemente amplia de aspecto similar a la verruga plantar, la histología típica es una invasión local mínima con baja incidencia de metástasis y buen pronóstico. Para el diagnóstico correcto, tanto el examen físico y el complementario debe ser exhaustivo y realizado mediante biopsia quirúrgica. La resección radical es el tratamiento de elección con confirmación histológica de los márgenes de resección libres del tumor.

Conclusiones: El carcinoma verrucoso plantar puede presentar dificultad diagnóstica, la resección quirúrgica completa de la lesión permite el diagnóstico, presenta un buen pronóstico y pronta curación.

Palabras clave: carcinoma verrucoso; epitelioma cuniculatum; carcinoma de células escamosas; tumor. 


\section{ABSTRACT}

Background: Verrucous carcinomas rarely mentioned in the literature its location on the soles of feet, being a distinct variant of squamous cell carcinoma seen in mucosa and skin, including the feet.

Objective: To describe the clinical, histopathological and laboratory and clinical results of an unusual case of a verrucous carcinoma on the sole.

Methods: We present a rare case of a verrucous carcinoma in the left sole, at the first metatarsal, which was completely resected and remained without evidence of tumor recurrence or metastasis during follow-up over a period of four years. On this basis, we reviewed the published cases of verrucous carcinoma in the foot, to define the characteristics of this rare tumor and give diagnostic and therapeutic recommendations.

Results: Verrucous carcinoma is characterized by a slow but inexorable clinical presentation similar in appearance to large plantar wart, histology is typical minimal local invasion with low incidence of metastases and good prognosis. For the correct diagnosis, both physical examination and must be comprehensive and complementary performed by surgical biopsy. Radical resection is the treatment of choice with histological confirmation of free resection margins of the tumor. Conclusions: The plantar verrucous carcinoma may present diagnostic difficulty, complete surgical resection of the lesion allows the diagnosis, prognosis and has good early healing.

Key words: verrucous carcinoma; cuniculatum epithelioma; squamous cell carcinoma; tumor.

Sumario: 1. Introducción. 2. Caso clínico. 3. Discusión. 4. Conclusiones. Bibliografía.

Referencia normalizada: López López, D., Rodríguez Sanz, D., Morales Ponce, Á., Soriano Medrano, A. Carcinoma verrucoso plantar. A propósito de un caso poco frecuente. Rev. Int. Cienc. Podol. 2013; 7(2): 83-88. 


\section{INTRODUCCIÓN}

El carcinoma verrucoso (CV) es una forma particular de carcinoma epidermoide que fue descrito por primera vez por Ackerman en 1948 en la cavidad oral, se caracteriza por estar bien diferenciado, por una evolución lenta y un grado bajo de malignidad después de la exéresis quirúrgica ${ }^{1,2,3}$.

El inicio de la lesión puede aparecer en cualquier parte del cuerpo y dependiendo del lugar de asentamiento existen tres tipos: el carcinoma verrucoso de aparato digestivo, el de la zona genital y el cutáneo que es el tipo de carcinomas que se pueden encontrar a nivel del pie ${ }^{4,5}$.

Este tumor es relativamente raro y generalmente suele aparecer en la etapa adulta, con predominancia en la sexta década de la vida y en varones blancos con un porcentaje de casos del $85 \%^{6,7,8}$.

El carcinoma verrucoso es probablemente causado por el VPH y se asocia más con los tipos del VPH 6 y VPH 11 y se cree que representa una lesión intermedia en un continuo desde lo menos patológico la verruga a los más invasivo el carcinoma de células escamosas ${ }^{9,10}$.

Los factores que favorecen la aparición de este tumor son habitualmente los traumatismos repetitivos, las quemaduras y la inflamación crónica relacionada con la presencia de úlceras en la pierna ${ }^{11,12,13}$.

La localización cutánea más frecuente se relaciona con los pies y especialmente en las plantas, a nivel proximal otras localizaciones

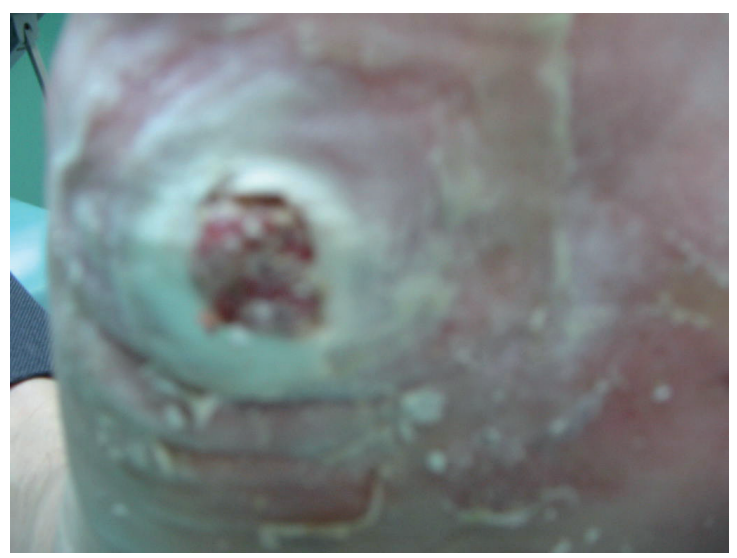

Figura 1. Carcinoma verrucoso plantar en el pie izquierdo. usuales son el tobillo, las piernas, los glúteos, el tronco y la cara ${ }^{14,15}$.

La clínica está relacionada con el estadío de la lesión, siendo el diámetro aproximado entre 2-20 cm, la apariencia la de una lesión verrucosa, queratósica, exofítica y cuando se localiza en zonas de presión la presentación es endofítica. La morfología recuerda por la coloración blanquecina, los surcos, las grietas e irregularidades de la piel a una coliflor ${ }^{16}$ (Figura 1).

El examen histológico de estas lesiones muestran una proliferación regular epitelial malignizada muy bien diferenciada y combinada con acantosis, hiperqueratosis e hiperpapilomatosis. Además, los canales epiteliales invaden hacia la dermis, sin irrumpir otros tejidos. La membrana basal es continua, sin anomalías celulares y las mitosis son normales. La dermis es fibrosa y los infiltrados celulares polimórficos son intradérmicos ${ }^{17,18,19,20}$. La confirmación histológica necesita realizar una biopsia profunda sobre la totalidad del tumor y de las proliferaciones escamosas hechas sin atipia de los queratinocitos que contienen en su centro un absceso con presencia de polimorfonucleares neutrófilos ${ }^{21}$.

La revisión de la literatura muestra la confusión y un potencial retraso en el diagnóstico y el tratamiento, haciendo hincapié en la gran importancia del examen histopatológico para su diferenciación de otras entidades clínicas como el carcinoma de células escamosas, carcinoma cuniculatum y otras entidades clínicas $^{22}$, siendo el objetivo de este caso clínico describir las características clínicas, histopatológicas y de laboratorio y los resultados de un caso clínico inusual de un carcinoma verrucoso en la planta del pie.

\section{CASO CLÍNICO}

Un hombre de 63 años de edad, acude a consulta, por primera vez en enero de 2008 con una lesión que no cicatrizaba, presentando una úlcera indolora en la superficie plantar del pie izquierdo en la zona del primer metatarsiano, que aumentó lentamente de tamaño durante los últimos 12 meses.

En la anamnesis no recuerda recibir un trauma previo, ni usar calzado ajustado y tampo- 
co suele utilizar los vestuarios públicos. En la historia clínica destaca la presencia de diabetes mellitus tipo II, de mas de diez años de evolución controlada con hipoglucemiantes orales y con dieta, sin presentar con anterioridad úlceras en los pies y tampoco haber trabajado en ambientes con exposición de los pies a irritantes químicos.

El examen físico revela pulsos tibiales intactos y una lesión ulcerada y móvil de $1,8 \times 0,9 \times$ $0,15 \mathrm{~cm}$ en la base del primer metatarsiano del pie izquierdo y las pruebas complementarias: las radiografías en carga lateral y dorsoplantar y la resonancia magnética no evidenciaron la existencia de ningún compromiso óseo. (Figura 2).

Los antecedentes de la enfermedad se relacionan con una biopsia de la lesión en un centro ambulatorio, en donde el servicio de anatomía patológica informa que se trataba de una verruga plantar.

Durante este periodo el tratamiento que le realizaron consistió en aplicación de 8 sesiones de acido nítrico ( 2 veces por semana durante 2 meses). A continuación 2 sesiones de podofilino (1 vez al mes). El siguiente tratamiento pautado consistió en aplicación de imiquimod (3 veces semanales durante un mes) y el tratamiento actual se relacionaba con curas oclusivas diarias con vaselina salicílica al $30 \% \sin$ conseguir durante el periodo de tratamiento

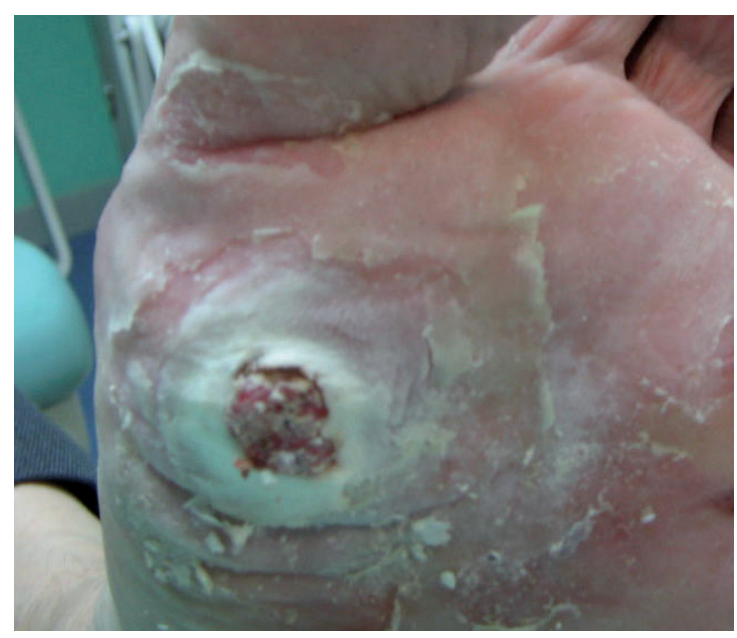

Figura 2. Aspecto inicial de la tumoración en el pie izquierdo mejora clínica y aumentando lentamente el tamaño de la lesión tumoral.

Después de un tratamiento preoperatorio, el paciente fue sometido a resección de la lesión, con márgenes claros y de espesor total, siendo los hallazgos anatomopatológicos finales consistentes con carcinoma verrucoso y la descripción del examen microscópico que corresponde a un engrosamiento y proliferación neoplásica escamosa de bajo grado de la epidermis. Además, la epidermis muestra acantosis, pliegues con colecciones de leucocitos neutrófilos $\mathrm{y}$ anchas y desiguales prolongaciones de crecimiento expansivo de la epidermis subyacente. Los queratinocitos aparecen bien diferenciados con núcleos regulares y pequeñas colecciones de queratina y mitosis numerosas, algunas de ellas atípicas. Los márgenes quirúrgicos de la pieza están libres de lesión (Figura 3).

El tratamiento postoperatorio consistió en curas diarias con sulfiadazina argéntica al $1 \%$ genérico durante un periodo de 8 semanas (Figura 4).

A continuación se planificaron revisiones al mes, tres meses y 6 meses durante el primer año de la exéresis del tumor y posteriormente se efectuaron revisiones cada 6 meses hasta la actualidad. El paciente evidencia un buen estado de salud, mostrando satisfacción y una actitud positiva, careciendo de recurrencia de la lesión.

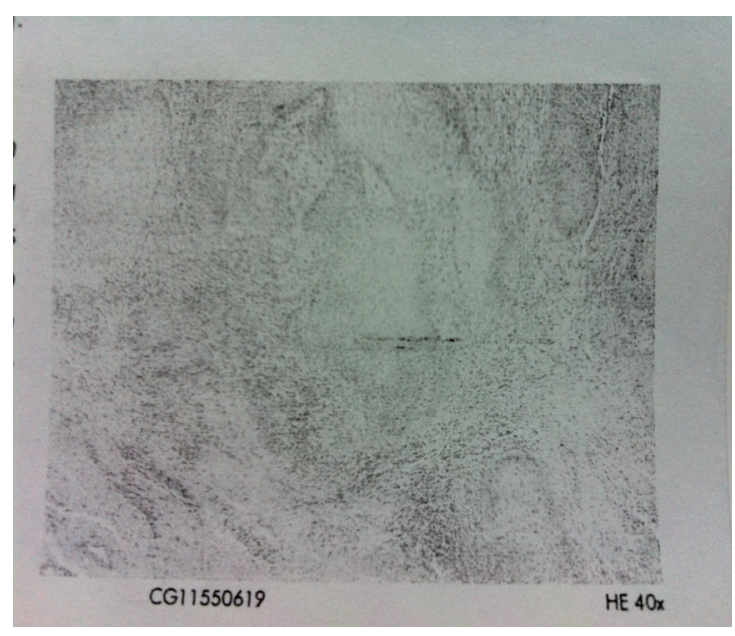

Figura 3. Acantosis irregular que se extiende por la epidermis y con colección de lecucocitos neutrófilos (HE, x40) . 


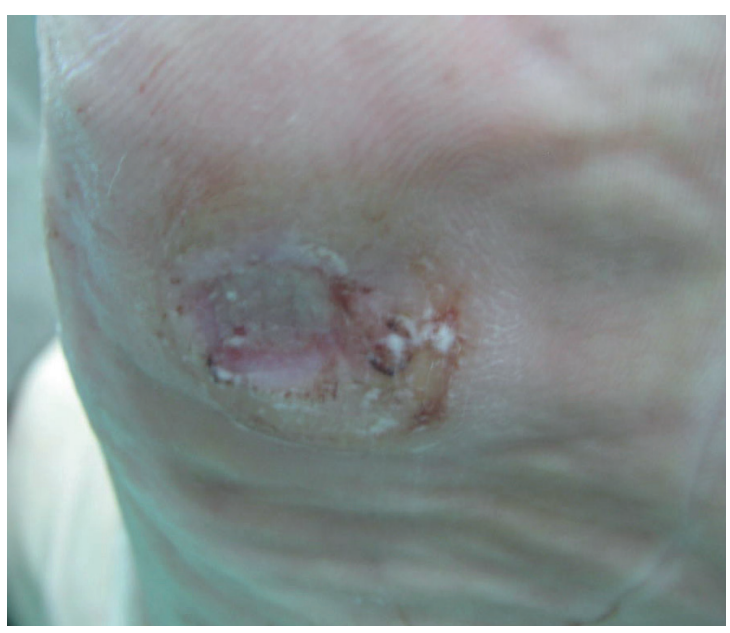

Figura 4. Aspecto del pie izquierdo luego de 3 semanas de la intervención quirúrgica .

\section{DISCUSIÓN}

Los carcinomas verrucosos está bien documentados en la literatura dental, cirugía dental y en la dermatológica pero existe una carencia de documentación a nivel del pie $^{23}$.

El concepto clínico y patológico del carcinoma verrrucoso se originó en 1948, cuando Lauren V. Ackerman describe una neoplasia de la cavidad oral en 31 pacientes, que posteriormente se describió en otras localizaciones como los pies $^{24,25,26}$

Las características clínicas la relacionan con un lento crecimiento y una invasión con displasia mínima, y biológicamente con una baja incidencia y grado bajo de metástasis ${ }^{27,28,29,30}$.

La causa del carcinoma verrucoso plantar no está clara por un lado se relaciona con el virus del papiloma humano y los tipos 1 a 4, 6, 11, 16 , y 18 que han sido relacionados en la patogénesis con la alteración de la actividad del on- cogen $\mathrm{p} 53^{31,32}$ y por otro lado se relaciona con infecciones virales, trauma e irritación a largo plazo $^{10,11,12}$.

El caso presentado de un hombre de 63 años de edad con antecedentes de diabetes mellitus, se ajusta al perfil típico de pacientes con carcinoma verrucoso dada su edad, sexo y el lugar habitual de localización que en el $53 \%$ de casos se presenta en el primer metatarsiano de la planta del pie. Además el aspecto de la lesión ulcerada papilomatosa verrucosa, con infección e irritación a largo plazo de la zona se adecuan con los datos encontrados en la literatura revisada ${ }^{8}$.

La recomendación diagnóstica y terapéutica de este tipo de tumores cutáneos se relaciona con la exéresis quirúrgica completa con los márgenes claros de la lesión del espesor to-

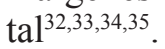

\section{CONCLUSIONES}

La presentación de este raro caso de carcinoma verrucoso, surge debido a la dificultad diagnóstica que puede presentar y la escasez de literatura asociada con la presentación en el pie. La posibilidad de malignización se asocia al cambio de la morfología y, aumento del tamaño del tumor siendo la resección quirúrgica completa de la lesión la que permite el diagnóstico, un buen pronóstico y pronta curación.

El tumor que se describe en este caso fue inusual en muchos aspectos, ya que originalmente se asocio, se trato con una forma compatible de verruga plantar, a pesar del hecho de que este tipo de tumor es común en varones y típicamente en las personas mayores siendo la exéresis completa de la lesión la que aporto luz a este extraño caso clínico.

\section{BIBLIOGRAFÍA}

1. Schwartz RA. (1995). Verrucous carcinoma of the skin and mucosa. J Am Acad Dermatol 1995; 32: 1-2.

2. Kaugars GE. Oral verrucous carcinoma, Oral Surg. Oral Med. Oral Pathol. Oral Radiol. Endod., 1999, 87, 268-269.

3. Bansal, M., Manchanda, K., Pandey, S.S. Verrucous cell carcinoma arising from an underlying giant keratoacanthoma. International Journal of Lower Extremity Wounds 2012; 11 (2). 85-87

4. Waskowska J, Koszowski R, Raczkowska-Siostrzonek A, Stemplewska K. Verrucous carcinoma of the tongue a rare case study Cent. Eur. J. Med. 2012; 7(2) 145-148. 
5. Alshahwan MA, Alghamdi KM, Alsaif FM. Verrucous Carcinoma Presenting as Giant Plantar Horns. Dermatol Surg 2007; 33:510-512.

6. Kao GF, Graham GH, Helwig EB. Carcinoma cuniculatum a clinicopathologic study of 46 cases with ulttrastrutural observations. Cancer 1992; 49: 2395-403.

7. Devaney KO, Ferlito A, Rinaldo A, El-Naggar AK, Barnes L. Verrucous carcinoma (carcinoma cuniculatum) of the head and neck: what do we know now that we did not know a decade ago? Eur Arch Otorhinolaryngol (2011) 268:477-480.

8. Lamchahab B, Guerrouj H, Bourra L, Marot F, Zouaidia O, Lamzaf M, et al. Aggressive course of intertoe verrucous carcinoma. Annales de Dermatologie et de Venereologie 2012; 139 (6-7) : 510-513.

9. Kelishadi SS, Wirth GA, Evans GR. Recalcitrant Verrucous Lesion Verrucous Hyperplasia or Epithelioma Cuniculatum (Verrucous Carcinoma) J Am Podiatr Med Assoc. 2006; 96 (2):148-53.

10. Ergün SS, Su O, Büyükbabaný N. Giant Verruca Vulgaris Dermatol Surg. 2004; 30(3):459-62.

11. Schell B, Rosen T, Rady P et al. Verrucous carcinoma of the foot associated with human Papilloma type 16. J Am. Acad Dermatol 2001; 45: 49-55.

12. Androphy EJ, Beutner K, Olbricht S. Human papillomavirus infection. In: Arndt KA, Robinson JK, le Boit PE, et al., eds. Cutaneous Medicine and Surgery: An Integrated Program in Dermatology. Philadelphia: W.B. Saunders, 1996:1100.

13. Lowy DR, Androphy EJ. Dermatology in General Medicine, 4th ed. New York: McGraw-Hill, 1993:2611.

14. Barnet JH, Estoa SA.. Multiple epithelioma cuniculatum in a multilating keratoderma. Cutis 1985; 35: $345-7$.

15. Schalock PC, Kornik RI, Baughman RD, Chapman MS. Treatment of verrucous carcinoma with topical imiquimod. J Am Acad Dermatol 2006; 54 (5): 233-235.

16. Barriere H, Vivion M. Epithelioma cuniculatum. Arch Dermatol 1986; 112: 1295-6.

17. Gayrard L, Cannilot S, Balme B et al. Epithelioma cuniculatum sur ulceration chronique du pied. Nouv Dermatol 1991; 10: 211.

18. Mekee PH, Wilkinson JD, Black MM. Carcinoma (epithelioma) cuniculatum a clinicopathologic study of nineteen cases and review of litterature. Histopathology 1981; 5: 425-36.

19. Bouquot J.E., Oral verrucous carcinoma. Incidence in two US populations, OralSurg. Oral Med. Oral Patho.l Oral Radiol. Endod., 1998, 86, 318-324.

20. Koch B.B., National survey of head and neck verrucous carcinoma, Cancer, 2001, 92,110-120.

21. Warshaw EM, Templeton SF, Washington CV. Verrucous carcinoma occuring in a lesion of oral lichen planus. Cutis 2000; 65: 219-22.

22. Kubik MJ, Rhatigan RM. Carcinoma cuniculatum: not a verrucous carcinoma J Cutan Pathol 2012; 1-5.

23. Rosales MA, Martin BR, Armstrong DG, Nixon BP, Hall HR. Verrucous hyperplasia: a common and problematic finding in the high-risk diabetic foot. Journal of the American Podiatric Medical Association 2006;96(4):348-50.

24. Assaf C, Steinhoff M, Petrov I, et al. Verrucous carcinoma of the axilla: case report and review. J Cutan Pathol 2004;31:199-204.

25. Ackerman LV. Verrucous carcinoma of the oral cavity. Surgery 1948;23:670-8.

26. McKay C, McBride P, James Muir J.Plantar verrucous carcinoma masquerading as toe web intertrigo. Australasian Journal of Dermatology 2012; 52(2): 20-22.

27. Kraus FT, Perez C. Verrucous carcinoma: clinical and pathologic study of 105 cases involving oral cavity, larynx and genitalia.Cancer 1966; 19: 26-38.

28. Wasserman PL, Taylor RC, Pinillia J, Wuertzer SD. Verrucous carcinoma of the foot and enhancement assessment by MRI. Skeletal Radiol. 2009; 38(4):393-395.

29. Zanini M. Verrucous carcinoma. Med Cutan Ibero. 2011;39(1):26-9.

30. Pilotti S, Donghi R, D'Amato L. HPV detection and p53 alteration in squamous cell verrucous malignancies of the lower genital tract. Diagn Mol Pathol 1993; 2: 248.

31. Pattee SF, Bordeaux J, Mahalingam M, Nitzan YB, Maloney ME. Verrucous carcinoma of the scalp. J Am Acad Dermatol. 2007; 56(3):506-7.

32. Mohs FE, Sahl WJ: Chemosurgery for verrucous carcinoma. J Dermatol Surg Oncol 1979; 5: 302.

33. Wolf H, Platzer P, Vécsei V. Verrucous carcinoma of the tibia arising after chronic osteomyelitis: a case report. Wien Klin Wochenschr. 2009; 121(1-2):53-6.

34. Wegener E. Glomus tumors of the nail unit: a plastic surgeon's approach. Dermatol Surg 2001; 27:240-1.

35. Garman ME, Orengo IF, Netscher D, Schwartz MR, Rosen T. On glomus tumors, warts, and razors. Dermatol Surg. 2003 Feb; 29 (2):192-4. 